\title{
BIOFUELS
}

\section{Finding the right blend}

Proc. Natl Acad. Sci. USA 116, $26421-26430$ (2019)

Lignocellulosic biomass is a source of renewable carbon and can be converted into molecules that are blendable with fossil fuels, such as diesel. These so-called blendstocks can improve the properties of the fuel while reducing its carbon intensity. However, they must satisfy a number of compatibility criteria and choosing which to target is challenging, given the large number of products that could potentially be synthesized from lignocellulose. Now, Derek $\mathrm{R}$. Vardon and colleagues across the USA computationally screen oxygenate molecules that can be derived from lignocellulose to identify those with appropriate properties and develop a catalytic process to synthesize a candidate molecule.

The researchers take short-chain carboxylic acids as a starting point and consider what molecules could be made from them. These carboxylic acids can be synthesized from waste biomass, but have poor fuel properties so cannot be used directly as blendstocks. The researchers predict the properties of around 50 different oxygenates spanning seven chemical families, evaluating fuel performance characteristics, deployment feasibility and health and safety aspects. From this analysis, they find that 4-butoxyheptane - $\mathrm{a} \mathrm{C}_{11}$ branched ether - should be promising as a blendstock. Through a continuous, solventfree multi-step catalytic synthesis, the researchers then produce 4-butoxyheptane at litre-scale and find that experimental fuel property measurements agree with their predictions in most cases. Moreover, when blended with diesel at $20 \mathrm{vol} . \%$, 4-butoxyheptane decreased the sooting tendency of the fuel by $20 \%$ and increased ignition quality by $10 \%$.

James Gallagher

Published online: 19 February 2020

https://doi.org/10.1038/s41560-020-0569-X 